\section{With a little help from glia}

Whether glia cells are involved in the induction of long-term potentiation (LTP) - a form of synaptic plasticity - in vivo remains controversial. Rusakov and colleagues now show that $\mathrm{Ca}^{2+}$-dependent $\mathrm{D}$-serine release from astrocytes regulates NMDAR ( $N$-methyl-D-aspartate receptor)dependent synaptic plasticity in acute hippocampal slice preparations from adult rats, providing evidence for such an involvement.

Co-agonists, such as D-serine and glycine, enable NMDAR activation by glutamate by binding to a co-agonist site of the NMDAR. Cultured astrocytes release $\mathrm{D}$-serine in a $\mathrm{Ca}^{2+}$-dependent manner, and

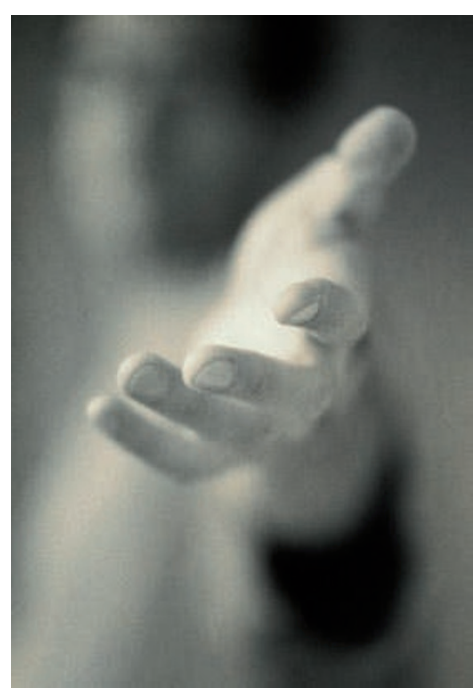

D-serine is required for LTP in culture. To test whether this also applies in a more physiological system - a long-standing controversy in the field - the authors patched individual astrocytes in acute hippocampal slices and monitored field excitatory postsynaptic potentials at synapses between Schaffer collaterals (SCs) and CA1 pyramidal cells. This experimental set-up allowed the authors to monitor and control the intra-astrocytic $\mathrm{Ca}^{2+}$ concentration $\left(\left[\mathrm{Ca}^{2+}\right]_{\mathrm{i}}\right)$ and to introduce pharmacological compounds to regulate D-serine synthesis.

High-frequency stimulation of the SCs increases astrocyte $\left[\mathrm{Ca}^{2+}\right]_{i}$. Blocking this increase (by clamping $\left[\mathrm{Ca}^{2+}\right]_{\mathrm{i}}$ ) suppressed LTP at nearby synapses, an effect that was rescued by extracellular addition of $\mathrm{D}$-serine. These results suggest that $\mathrm{Ca}^{2+}$ dependent release of an NMDAR co-agonist from astrocytes is essential for the induction of LTP.

When the authors blocked the synthesis of D-serine in individual astrocytes and depleted their D-serine pool by high-frequency stimulation, subsequent LTP induction was suppressed, providing direct evidence for a role of astrocyte-derived $\mathrm{D}$-serine in LTP induction.

Astrocytes can be connected by gap junction channels, raising the question of how far the influence of a single astrocyte and its associated network on synaptic activity extends into the surrounding area. The authors monitored LTP simultaneously in two neighbouring hippocampal areas that contained two astrocytes. Blocking the increase of $\left[\mathrm{Ca}^{2+}\right]_{\mathrm{i}}$ in one of the astrocytes suppressed LTP in synapses that were in reach of gap junction-connected astrocytes. Synapses in the vicinity of the other astrocyte, in which $\mathrm{Ca}^{2+}$ rises were allowed, were not affected, demonstrating that the synaptic effects of the two astrocytes could be independent.

This study elegantly demonstrated that the induction of LTP at hippocampal SC-CA1 synapses requires $\mathrm{Ca}^{2+}$-dependent release of $\mathrm{D}$-serine from astrocytes. Considering the density of neurons and astrocytes in CA1 in addition to gap junctionconnected astrocytes, D-serine released from a single astrocyte might affect synaptic plasticity of hundreds of neurons nearby.

Claudia Wiedemann

ORIGINAL RESEARCH PAPER Henneberger, C., Papouin, T., Oliet, S. H. R. \& Rusakov, D. A. Long-term potentiation depends on release of D-serine from astrocytes. Nature 463, 232-236 (2010)

FURTHER READING Giaume, C., Koulakoff, A., Roux, L., Holcman, D. \& Rouach, N. Astroglial networks: a step further in neuroglial and gliovascular interactions. Nature Rev. Neurosci. 11, 87-99 (2010) 